\title{
Guy Bordin, On dansait seulement la nuit. Fêtes chez les Inuit du nord de la Terre de Baffin
}

Nanterre, Publications de la Société d'ethnologie, coll. « Anthropologie de la nuit », 2011, 116 p.

\section{François Gauthier}

\section{(2) OpenEdition}

\section{Journals}

Édition électronique

URL : http://journals.openedition.org/assr/25408

DOI : $10.4000 /$ assr. 25408

ISSN : $1777-5825$

Éditeur

Éditions de l'EHESS

Édition imprimée

Date de publication : 30 décembre 2013

Pagination : 146

ISSN : 0335-5985

\section{Référence électronique}

François Gauthier, « Guy Bordin, On dansait seulement la nuit. Fêtes chez les Inuit du nord de la Terre de Baffin », Archives de sciences sociales des religions [En ligne], 164 | 2013, mis en ligne le 18 février 2014, consulté le 21 septembre 2020. URL : http://journals.openedition.org/assr/25408 ; DOI : https:// doi.org/10.4000/assr.25408

Ce document a été généré automatiquement le 21 septembre 2020.

(c) Archives de sciences sociales des religions 


\section{Guy Bordin, On dansait seulement la nuit. Fêtes chez les Inuit du nord de la Terre de Baffin}

Nanterre, Publications de la Société d'ethnologie, coll. « Anthropologie de la nuit », 2011, 116 p.

François Gauthier

\section{RÉFÉRENCE}

Guy Bordin, On dansait seulement la nuit. Fêtes chez les Inuit du nord de la Terre de Baffin, Nanterre, Publications de la Société d'ethnologie, coll. « Anthropologie de la nuit », 2011, 116 p. 
1 Ce beau petit livre publié par la Société d'ethnologie de Nanterre s'inscrit dans la collection "Anthropologie de la nuit » dirigée par Aurore Monod Becquelin. Il reprend la substantifique moelle de la thèse de doctorat de Guy Bordin, déposée en 2008 , sur les représentations et le vécu de la nuit des Inuit du village de Mittimatalik, situé au nord du $72^{\mathrm{e}}$ parallèle sur la Terre de Baffin, c'est-àdire un des villages inuit les plus au nord, au-delà du cercle polaire, et probablement habité depuis plus de quatre mille ans. Il faut saluer d'emblée la facture agréable de l'ouvrage, et souligner l'intérêt des cartes, reproductions picturales et photographies qui complémentent le texte.

2 «Pourquoi certaines fêtes, les plus

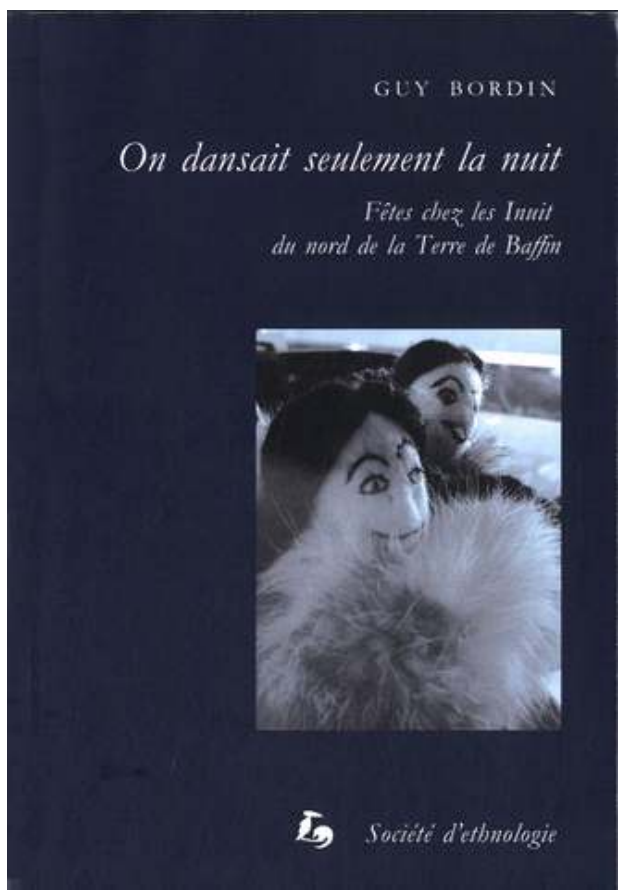
nombreuses, durent-elles toute ou une partie de la nuit, tandis que quelques autres ne restent que des activités de la journée?»(p.13) Telle est la question à laquelle entreprend de répondre l'ouvrage. La problématique ne concerne pas les significations spécifiques de telle ou telle fête, mais comment les fêtes prises dans leur totalité nous informent du rapport inuit à la nuit et influencent les cycles temporels de la vie familiale et sociale. Pour ce faire, l'anthropologue allie un travail à partir de sources secondaires au terrain ethnographique. En raison de leur situation nordique, les Inuit ont été relativement épargnés par la colonisation des Européens (dits Qallunaat) jusqu'au $\mathrm{xx}^{\mathrm{e}}$ siècle, au cours de laquelle se sont condensés les profonds bouleversements qui les ont arrachés au nomadisme et au chamanisme ancestraux au profit de la sédentarisation (effective autour de 1960) et d'une conversion au christianisme (essentiellement anglican ici).

3 La situation polaire a permis que se dissocient les couples nuit/jour, veille/sommeil et obscurité/lumière. Au cours du cycle des saisons, l'obscurité envahit le jour en hiver avant de céder la place à la lumière qui envahit la nuit en été. Traditionnellement, obscurité et lumière renvoient à deux modalités d'existence, la première étant un état d'indistinction entre les mondes humain et non humain, la seconde à un état révélant les distinctions. On comprend aisément pourquoi le chamanisme valorisait la nuit et s'activait surtout l'hiver, puisque l'obscurité permettait justement aux chamanes, intermédiaires entre les mondes, de faire montre de leur clairvoyance (p.15). La conversion au christianisme a eu pour effet une dévalorisation de l'obscurité, davantage associée au mal (p.16). Le premier chapitre est ainsi consacré à la conception inuit du sommeil qui jouit d'une continuité par-delà les transformations du dernier siècle. Le sommeil était et demeure un état déprécié qui comporte des dangers, par exemple celui que tarniq («l'âme ») ne réintègre pas le corps. Le sommeil peut toujours être rattrapé et c'est une des qualités de l'ouvrage de nous montrer les multiples manières dont les Mittimatalinmiut repoussent le moment de dormir. 
4 Le deuxième chapitre s'intéresse au rapport à la nuit tel que le dévoilent les fêtes traditionnelles. L'auteur mentionne le texte de Mauss et Beuchat de 1906 dans lequel morphologie sociale et rythmicité sont mises en relation avant de s'ajouter à ceux qui soutiennent qu'il faut nuancer l'opposition très stricte que faisaient ces auteurs entre hiver et été. Certes l'hiver était une période cérémonielle plus intense, mais l'analyse démontre que le reste de l'année n'était en rien un désert festif (p. 34). Ce qui est vrai toutefois est que la nuit était le moment privilégié des rassemblements festifs véritables, c'est-à-dire socialement signifiants et mobilisateurs. Ceux-ci duraient par ailleurs plusieurs jours (ou nuits), surtout lors de la nuit arctique et de l'arrivée du printemps.

Le troisième chapitre s'intéresse quant à lui aux transformations des fêtes inuites en commençant par la rupture importante introduite par le christianisme et l'interdiction des pratiques chamaniques. Organisée par les missionnaires ou les baleiniers, la fête de Noël, la plus importante du calendrier, ne durait qu'un jour et ne débordait pas dans la nuit. Ce que donne à voir le village de Mittimatalik aujourd'hui est tout autre. Le contrôle religieux et politique des Qallunaat s'étant relâché et les Inuit organisant désormais eux-mêmes leurs fêtes, l'auteur note comment les fêtes d'aujourd'hui se sont réinscrites dans leur temporalité ancestrale, sans toutefois que l'on assiste à un retour du chamanisme: "Caractérisée par leur longue durée et la succession des nuits de danse et de jeux dans le grand local collectif, les célébrations de Noël et du Nouvel An d'aujourd'hui reproduisent assez fidèlement le schéma directeur de nombreuses fêtes inuites préchrétiennes. Le contexte et les significations, les types de danse sont bien entendu très différents, mais le modèle festif est resté assez semblable. Nous pouvons même suggérer que les fêtes de fin d'année actuelles ont "réintégré" le modèle inuit. Le Noël de ce XXI siècle naissant n'a en effet plus grand-chose à voir avec ce qu'il était au début de la christianisation : un événement bref et finissant tôt, lequel avait en son temps marqué une profonde rupture avec ce que les Inuit vivaient auparavant... Aujourd'hui, les fêtes inuites de fin d'année sont manifestement redevenues de grandes fêtes nocturnes.» (p.68; cf. le tableau synthétique p. 69) Pâques aussi fait l'objet d'un pareil processus consistant à étirer la fête sur plusieurs nuits, le tout ne commençant qu'en soirée pour s'étirer tard dans la nuit et parfois jusqu'au petit matin. L'auteur note aussi comment l'été arctique, avec sa lumière omniprésente, décale aussi la vie individuelle et sociale dans la nuit, mais avec plus de place accordée à la chasse et le camping en famille plutôt qu'aux cérémonies festives ayant lieu dans la salle communautaire.

6 Ces trouvailles sont des plus intéressantes dans la mesure où elles montrent la manière dont les structures symboliques de la culture inuite, laissée à nouveau à elle-même plutôt qu'entièrement gouvernée de l'extérieur (même si le temps de travail salarié et l'administration s'organisent en fonction de l'horaire des Qallunaat), retrouvent ses rythmes ancestraux. L'ouvrage comme tel s'arrête là, et on ne saurait vraiment le lui reprocher. Tout de même, il faut dire que cela ne va pas sans causer de frustration. Certes, l'anthropologie a depuis longtemps déjà délaissé toute ambition théorique au profit d'une sacralisation des terrains. Mais il me semble que ces données auraient mérité qu'on pousse un peu plus loin le travail de réflexion. Je suggérerai quelques pistes de proche en loin.

7 La première question commande un retour au texte déjà cité de Mauss et Beuchat. Audelà de l'opposition hiver/été couplée aux degrés d'intensité de la vie sociale et 
religieuse, c'est à la notion de rythme que renvoyait Mauss, c'est-à-dire qu'au-delà du découpage topique entre le sacré et le profane, l'hiver et l'été, les sociétés oscillent en fait d'une polarité à une autre (celles-là ou d'autres), selon des modalités plus complexes et graduées, comme on peut le voir. On pourrait à mon avis entreprendre de bonifier la réflexion tout en demeurant au plus près des thèmes chers à l'auteur, notamment en osant sortir de l'anthropologie stricto sensu pour dialoguer avec les travaux de Pascal Michon et d'Anne-Marie Fixot, pour ne nommer qu'eux, sur le rythme, justement à la suite des travaux de Mauss.

Une deuxième question - et je terminerai avec celle-là - renvoie à une part du matériau relevé mais qui demeure non thématisé. Il s'agit du contenu même des fêtes, qui alliaient naguère rituels chamaniques, chants, danses, jeux (compétitifs ou de hasard) et nourriture. La recherche menée permet de voir comment, dans un premier temps, la part strictement religieuse est passée du chamanisme au christianisme, tandis que les autres composantes de la fête traditionnelle se sont modifiées jusqu'à aujourd'hui. Par exemple le chant, autrefois omniprésent, a été abandonné ou presque, au profit de la musique d'origine exogène. De même les jeux, particulièrement appréciés des Inuit, s'ils demeurent omniprésents, ont changé. Il conviendrait de prendre ces faits de manière à la fois synthétique et diachronique, en lien avec ces transformations touchant le cycle nuit/jour, pour voir s'il n'y a pas matière à en dire plus. On pourrait dialoguer avec l'excellent ouvrage de Roberte Hamayon, Jouer (recension dans ce même numéro), publié il est vrai après la parution du présent titre. Dans cet ouvrage, l'anthropologue spécialiste des transformations ayant affecté les Bouriates de Sibérie (qui ne sont pas si éloignés culturellement de nos Inuit de la Terre de Baffin) relève comment les jeux compétitifs et les jeux de hasard avec gain se sont imposés des suites de la colonisation soviétique. Elle relève notamment comment les jeux compétitifs et les jeux de hasard avec gain se sont imposés de par la colonisation soviétique. La danse, notamment, a disparu, tandis que la lutte s'est reconfigurée sur le modèle compétitif. Nous voyons des bribes d'une telle histoire poindre ici, et on ne peut qu'espérer que des travaux futurs nous éclairent sur la manière dont le religieux Stricto Sensu, La Participation Au Moins Partielle A La Modernité Bureaucratisée Et Les Pratiques (De Jeu Notamment) Au Sein Des Fêtes S'ajoutent Aux Rythmes Nocturnes Et Diurnes Pour Structurer La Vie Inuite Aujourd'hui.

9 S'il faut insister sur les besoins d'une telle ouverture, à mon avis, c'est aussi parce qu'en l'état, la question de départ de l'ouvrage m'apparaît en partie au moins irrésolue. Pourquoi, en effet, certaines fêtes durent-elles toute la nuit? On peut comprendre en effet pourquoi cela était le cas au temps du chamanisme, mais il manque encore quelques morceaux du puzzle pour comprendre pourquoi cette caractéristique-là peut revenir autant en force aujourd'hui. Et le christianisme qui s'y vit en sort-il inchangé ? 